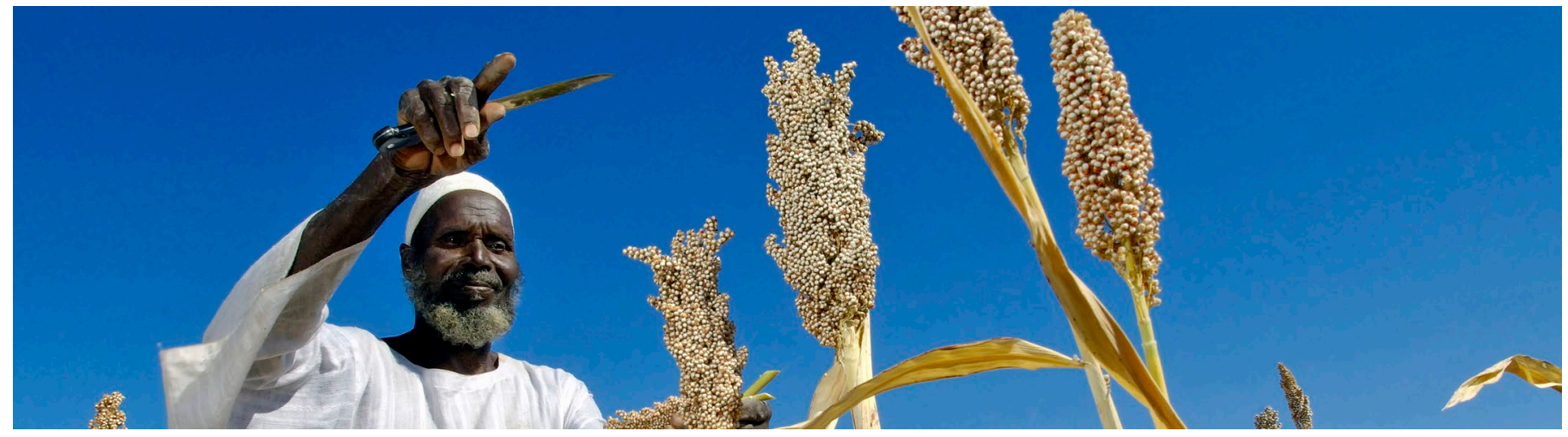

\title{
Food System Resilience Assessment
}

\begin{abstract}
A practical approach to capture how specific system dynamics shape food and nutrition outcomes in protracted crises. Version 1.0
\end{abstract}

Charleen Malkowsky and Gerrit-Jan van Uffelen

\section{Introduction: the need to put food system resilience into practice}

"The magnitude and severity of food crises worsened in 2020 as protracted conflict, the economic fallout of COVID-19, and weather extremes exacerbated pre-existing fragilities." ${ }^{1}$

Hunger is on the rise again, especially in protracted crises, and throughout the Covid-19 epidemic, the concept of food system resilience is gaining more traction and popularity as the search for new solutions intensifies in order to address increases of food insecurity.

The Global Report on Food Crises (GRFC) emphasises that humanitarian aid is not the answer to solving hunger, but that a more systemic nexus approach is needed to address root causes of food crises and hunger. While concepts and theories on food system resilience, especially for stable environments, are developing rapidly with many publications emerging everywhere, there are still only very few examples of what the concept means in practice - particularly in the context of protracted crises where new solutions are needed the most. There is a risk that the concept of food system resilience remains a high-level discussion whilst not being instrumentalized to context-specific programming realities and, thus, it is important to focus on operationalising the Food System Resilience into a more concrete and practical approach for guiding field work and interventions in local protracted crises realities.

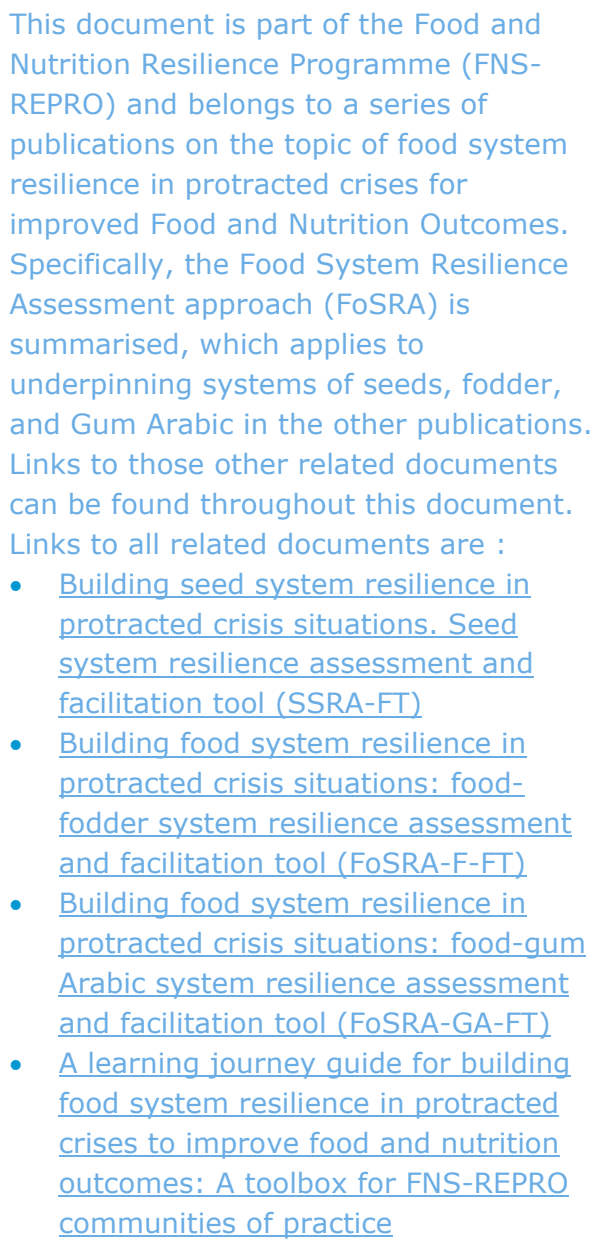

This document is part of the Food and Nutrition Resilience Programme (FNSREPRO) and belongs to a series of publications on the topic of food system resilience in protracted crises for improved Food and Nutrition Outcomes. Specifically, the Food System Resilience Assessment approach (FoSRA) is summarised, which applies to underpinning systems of seeds, fodder, and Gum Arabic in the other publications. Links to those other related documents can be found throughout this document. Links to all related documents are :

- Building seed system resilience in protracted crisis situations. Seed system resilience assessment and facilitation tool (SSRA-FT)

- Building food system resilience in protracted crisis situations: foodfodder system resilience assessment and facilitation tool (FoSRA-F-FT)

- Building food system resilience in protracted crisis situations: food-gum Arabic system resilience assessment and facilitation tool (FoSRA-GA-FT)

- A learning journey guide for building food system resilience in protracted crises to improve food and nutrition outcomes: A toolbox for FNS-REPRO communities of practice

1 This fifth annual Global Report on Food Crises (GRFC 2021) results from months of collaboration among numerous members of the international humanitarian and development community. 


\section{Analysing Food System's Interface with Specific Target Systems \& FNS Outcomes}

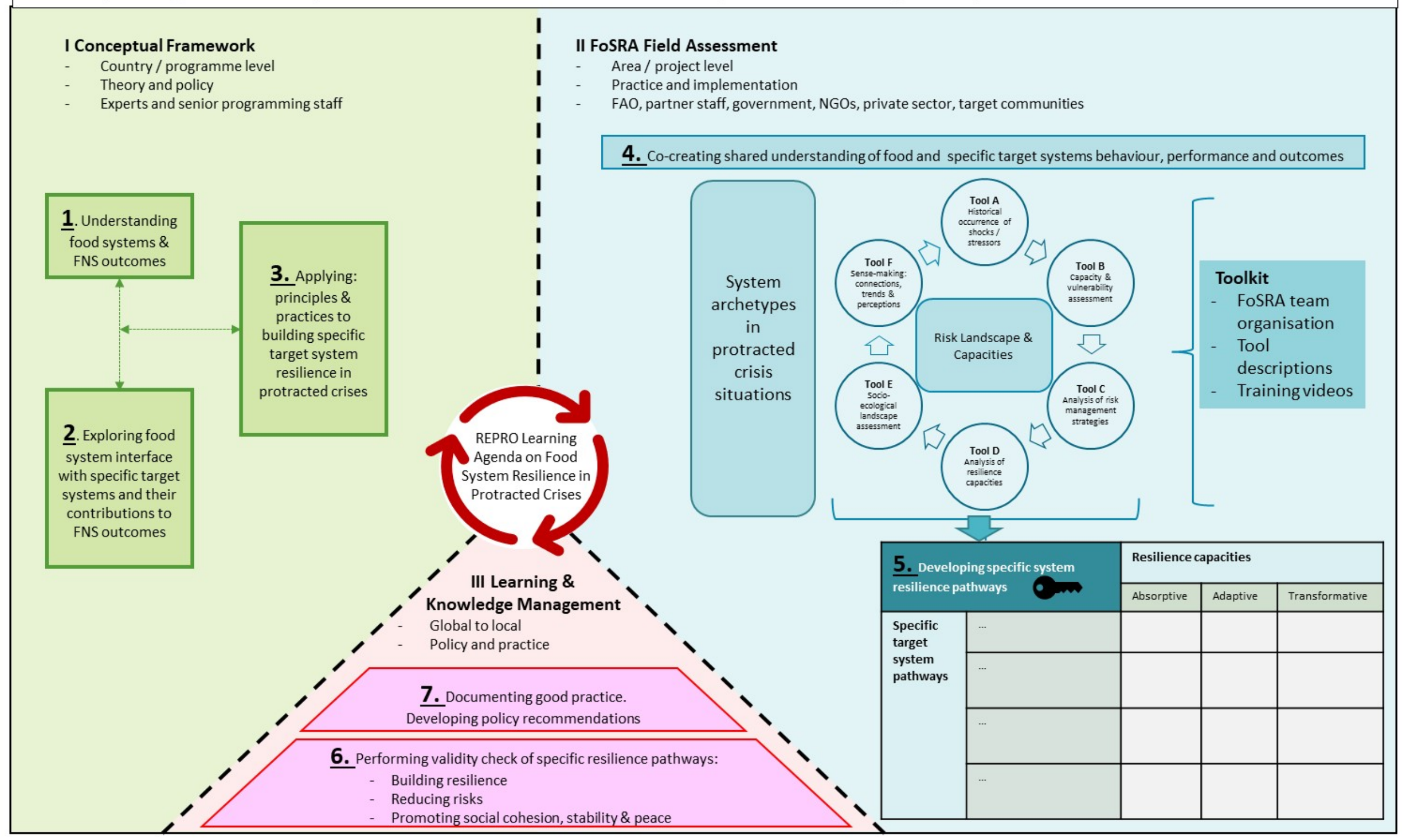


This paper provides a summary of the 'Food System Resilience Assessment (FoSRA)' approach, which is designed for analysis and planning of interventions aimed at addressing food insecurity in protracted crisis contexts. The approach gives practical meaning to the concept of food system resilience by viewing the food system in relation to a specific value chain or target system through capturing several different actor levels perspectives: from local populations and national organisations to governments \& global institutions.

The FoSRA approach has been developed by Wageningen Centre for Development Innovation (WCDI), in close consultation with the Food and Agricultural Organisation of the United Nations (FAO), to develop context-specific food system resilience pathways for selected areas in Sudan, South Sudan, and Somaliland as part of the Food and Nutrition Security REsilience PROgramme (FNS-REPRO, hereafter: REPRO) and can be seen as a zero draft that will be further validated and updated as the project proceeds. While testing and validation of this approach focus on the specific target systems of REPRO (Gum Arabic, livestock fodder and seeds), it is assumed that the approach is also a valuable entry point for assessing other specific value chain systems, so long as it is adapted and contextualised accordingly.

This paper is structured along the FoSRA frameworks' parts: I. the conceptual framework; II. the FoSRA field assessment and III. learning and knowledge management. Each part elaborates in further detail on its respective component therein. Finally, the last section highlights some concluding remarks.

\section{Part: Conceptual Framework}

Part one is the conceptual framework (left green part of the framework) and is conducted at a country and programme level with a focus on theory and policies. Stakeholders involved are experts on food systems or the specific target system and senior programming staff. This part I consists of three components:

1. Understanding the food system and how it produces Food and Nutrition Security (FNS) outcomes

2. Exploring the food system interface with a specific target system and its subsequent outcomes

3. Applying principles and best practices to building resilience of the food-and target system

The first component of part one analyses the overall food system. This is done by applying the Food System Framework (developed by van Berkum et al.) in the form of a quick scan. It assesses food production activities in the light of their interaction with socio-economic- and environmental drivers to create an understanding how FNS outcomes are produced.

The required data can be generated through several ways, such as expert interviews, a literature review (including sources such as fews.net, ICP info, the food system dashboard or FSIN) or by accessing the available data of relevant local and international institutions.

The second component of part one explores the food system interface with a specific target system. A specific target system can be any relevant value chain that interfaces with the food system, such as a seed system, a fodder system (which underpins the livestock system) or a Gum Arabic system (see hyperlinks for specific examples). A target system does not need to be a food producing system per se but can also be an income generating value chain, consequently impacting food security through increased purchasing power. The goal is to understand how this specific target system underpins the functioning of the food system and its subsequent FNS outcomes. This also serves as a rationale for selecting a specific target system when designing targeted interventions based on its promising interface and potential for improvement. 


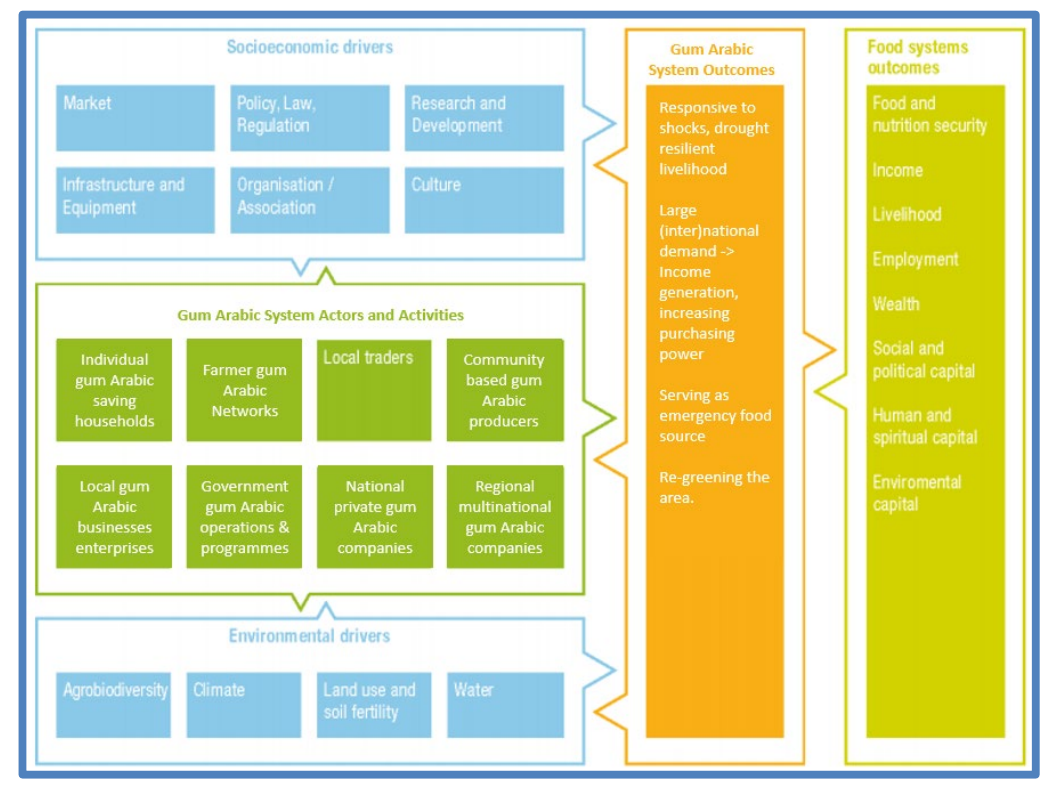

1 Example Understanding Gum Arabic systems as underpinning the food system

For this, more expert consultations and desk studies need to be conducted. A framework that conceptualises this interface was developed based on the food system framework and can be adapted flexibly to fit the specific target value chain systems' dynamics (see example box 1 for the case of Gum Arabic systems).

The third component of part one focusses on understanding the protracted crisis context by looking at its characteristics and dynamics, as well as resulting limitations and constraints on programming, conceptual and institutional levels. For this, we use the well-known work of Maxwell et al. from 2011. Moreover, the principles and practices for strengthening resilience for FNS in protracted crises, as developed by the Rome-based agencies (RBA), are applied. On this basis, draft guiding principles for the specific target system are developed. Furthermore, the resilience concept is being operationalised through a multi-stakeholder process during which different actors answer the key questions for building resilience: 'Resilience of what?', 'Resilience to what?', 'Resilience for whom?' and 'Resilience 'through what'?'. These, often varying, answers help build a common ground and understanding between stakeholders, as well as lending the concept of resilience meaning within the specific context for it to be operationalized to a practical programme (see 2 example box). This can help at a later stage to reduce trade-offs when designing programmes, as well as ensuring programming along the Humanitarian-Development-Peace nexus (hereafter: HDP nexus) since it facilitates a productive dialogue among food system actors. Finally, on this basis, the system boundaries and archetypes are defined.

In the case of REPRO Somaliland, this would be the resilience of pastoralist systems and respective connected livelihoods ('of what') towards key shocks and stressors such as droughts, deforestation, and conflicts ('to what') for (agro)-pastoralist households in Sool and Sanaag ('for whom') through the development of a partially commoditised \& supportive feed and fodder system ('through what').

However, the answers to those questions are likely to differ, depending on which of the varying actor groups is being asked - local beneficiaries, policy makers, or international experts. It is important to keep this in mind to mitigate any tensions caused by misunderstandings, and to create a dialogue to facilitate communication with one another in a transparent \& productive manner. This also creates an organic starting point for programming decisions.

2 Example Operationalising resilience in the specific case of food-fodder system interfaces 
The second part (blue part of the framework on the right) is conducted on an area-based or project level, looking at practice and implementation. Actors involved vary, ranging from governmental staff, NGO workers, local community members (including women and youth), private sector actors or local staff of global institutions such as FAO. The fourth and fifth components form part II:

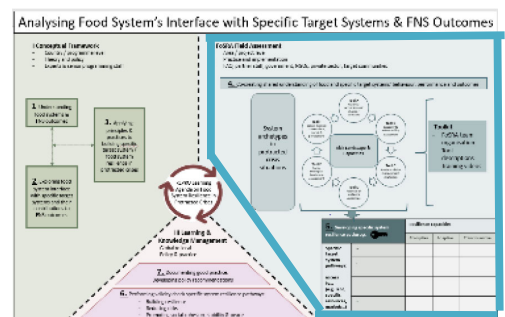

4. Co-creating a shared understanding of food- and specific target systems' behaviours, performances, and outcomes

5. Developing specific system resilience pathways

The fourth component builds upon a range of different tools that are used within the geographical area of interest. These can be flexibly adapted or replaced to make them most relevant to the specific target system. The basic tools include:

A. a historical timeline of shocks and stressors affecting the system;

B. a capacity and vulnerability assessment;

C. an analysis of existing risk management strategies;

D. an analysis of resilience capacities;

E. an assessment of the socio-ecological landscape and

F. a process of sense-making through key informant interviews.

Depending on the type of target system one selects, the tools can be shaped specifically to the target system (as the seed system example in example box 3 illustrates) or take a more general livelihood/ food security perspective. There is also an elaborate facilitation kit for this step, explaining in detail how these tools should be best implemented (based on the examples of seeds, gum arabic and fodder).

Tools for a seed systems resilience assessment:

a. Analysis of Crop Diversity, Availability, and Preference.

b. Analysis of Climate Resilient Crops and Varieties.

c. Social seed network analysis.

d. Seed Systems analysis.

e. Seed value chain analysis.

3 Example Specific tools to analyse seed systems

The fifth component develops the specific system resilience pathways within a programmes sphere of influence. These pathways can be imagined along the line of absorptive, adaptive and transformation resilience pathways vis-à-vis specific access dynamics, for example access to resources such as (fertile) land, existing trees and/or markets, or formality levels of a sector (formal, intermediate, informal).

With the input of previous components, this component makes sense of the specific target systems' behaviour in underpinning food systems' functioning and acknowledges that different segments of society benefit differently from potential changes and ensures that trade-offs are minimised. It is intended to develop several pathways centred around the identified leverage points, to allow for meaningful engagement of several segments of society to not only improve FNS outcomes but also reduce local tensions through increasing social cohesion. 


\section{Part: Learning and Knowledge Management}

The third part (red, bottom part of the framework) crosses all levels from local to global, looking jointly at policy and practice as related to food system resilience in protracted crises. The team of leading actors leading actor team of the FoSRA facilitates this step but, depending on the insights gained, all other types of actors may also play a role; specifically relevant local and global knowledge and research institutes. Part three consists of the last two components:

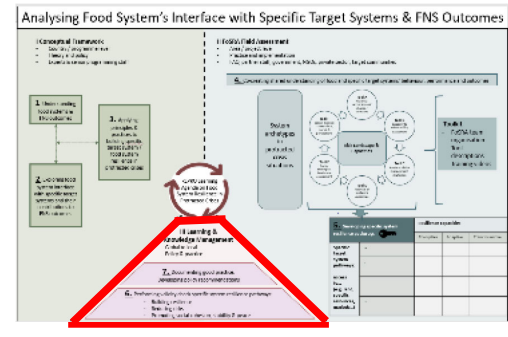

6. Validating the specific resilience pathways

7. Documenting good practice and policy recommendations

The sixth component is a validation process that can make use of one of three different methodologies, or a combination of those: a list of characteristics of a resilient community that describes the ideal situation and can be flexibly adapted through indictor selection; a nexus question list (to check if the pathways are sound from a humanitarian, developmental as well as peacebuilding perspective); and/or an adapted version of validating seed system pathways.

The last component focuses on contributing to a wider evidence-base for building resilient food systems by documenting best practices and developing policy recommendations on this basis. Useful questions for this step are: What were the most insightful learnings of the assessment/ action? How should these learnings be documented to create a reliable evidence base for future programmes and governance decisions? Are policies and practices logically aligned or support each other? Are there gaps between policy and desirable practice? How can policies adapted to reinforce good practices? How can these insights be useful in other contexts in the region or even globally?

\section{Concluding Remarks}

The FoSRA approach allows for the concept of food system resilience to be put into practice and can be used in several ways: as a baseline study and a diagnostic tool, for planning and designing programmes, for adaptive management or applied research. By selecting a target value chain system, the FoSRA is focussed on specific elements and thus gives meaning to the concept within the specific context based on strategic opportunities and particular interest of the users.

In protracted crises the understanding of local perceptions, local realities, and how to strengthen existing capacities is key to building resilience since these developed over time and reflect the needs of the given situation. In the field, silos blur naturally and bring together perspectives of humanitarian, development and peace/ stability actors to indicate the food systems' real dynamics, behaviours, performances and creation of FNS outcomes.

Reflection and learning are central to the FoSRA approach because they build the basis for real evidence-based programming and adaptive management, both critical for programming in protracted crises, which does not only serve the immediate needs of the targeted beneficiaries, but also has the potential to address root causes of food crises. 
This report can be downloaded for free at https://doi.org/10.18174/555007 or at www.wur.eu/cdi (under publications).

(C) 2021 Wageningen Centre for Development Innovation, part of the Stichting Wageningen Research. P.O. Box 88, 6700 AB Wageningen, The Netherlands. T + 31 (0)317 486800 , E info.cdi@wur.nl,www.wur.eu/cdi.

\section{(cc) BY-NC}

The Wageningen Centre for Development Innovation uses a Creative Commons Attribution 4.0 (Netherlands) licence for its reports.

The user may copy, distribute and transmit the work and create derivative works. Third-party material that has been used in the work and to which intellectual property rights apply may not be used without prior permission of the third party concerned. The user must specify the name as stated by the author or licence holder of the work, but not in such a way as to give the impression that the work of the user or the way in which the work has been used are being endorsed. The user may not use this work for commercial purposes.

The Wageningen Centre for Development Innovation accepts no liability for any damage arising from the use of the results of this research or the application of the recommendations.

Wageningen Centre for Development Innovation supports value creation by strengthening capacities for sustainable development. As the international expertise and capacity building institute of Wageningen University \& Research we bring knowledge into action, with the aim to explore the potential of nature to improve the quality of life. With approximately 30 locations, 6,800 members (6,000 fte) of staff and 12,900 students, Wageningen University \& Research is a world leader in its domain. An integral way of working, and cooperation between the exact sciences and the technological and social disciplines are key to its approach. 